\title{
Mouse Ficolin B Has an Ability to Form Complexes with Mannose-Binding Lectin-Associated Serine Proteases and Activate Complement through the Lectin Pathway
}

\author{
Yuichi Endo, ${ }^{1}$ Daisuke Iwaki, ${ }^{1}$ Yumi Ishida, ${ }^{1}$ Minoru Takahashi, ${ }^{1}$ \\ Misao Matsushita, ${ }^{2}$ and Teizo Fujita ${ }^{1}$ \\ ${ }^{1}$ Department of Immunology, Fukushima Medical University School of Medicine, 1-Hikarigaoka, Fukushima 960-1295, Japan \\ ${ }^{2}$ Department of Applied Biochemistry, Tokai University, Hiratsuka, Kanagawa 259-1292, Japan \\ Correspondence should be addressed to Yuichi Endo, yendo@fmu.ac.jp
}

Received 3 October 2011; Accepted 8 November 2011

Academic Editor: Nobutaka Wakamiya

Copyright ( $) 2012$ Yuichi Endo et al. This is an open access article distributed under the Creative Commons Attribution License, which permits unrestricted use, distribution, and reproduction in any medium, provided the original work is properly cited.

Ficolins are thought to be pathogen-associated-molecular-pattern-(PAMP-) recognition molecules that function to support innate immunity. Like mannose-binding lectins (MBLs), most mammalian ficolins form complexes with MBL-associated serine proteases (MASPs), leading to complement activation via the lectin pathway. However, the ability of murine ficolin B, a homologue of human $\mathrm{M}$-ficolin, to perform this function is still controversial. The results of the present study show that ficolin B in mouse bone marrow is an oligomeric protein. Ficolin B, pulled down using GlcNAc-agarose, contained very low, but detectable, amounts of MASP-2 and small MBL-associated protein (sMAP) and showed detectable C4-deposition activity on immobilized $\mathrm{N}$-acetylglucosamine. These biochemical features of ficolin B were confirmed using recombinant mouse ficolin B produced in CHO cells. Taken together, these results suggest that like other mammalian homologues, murine ficolin B has an ability to exert its function via the lectin pathway.

\section{Introduction}

Ficolins $(\mathrm{FCN} / \mathrm{Fcn})$ are a family of proteins comprising a collagen-like and a fibrinogen-like domain [1] the latter binding specifically to $\mathrm{N}$-acetyl compounds such as $\mathrm{N}$ acetylglucosamine (GlcNAc) [2-4]. Three types of ficolin have been identified in humans: L-FCN $[1,5]$, M-FCN $[6,7]$, and H-FCN [8]. Transcripts for L-FCN and HFCN are mainly produced in the liver, and the proteins circulate as serum ficolins, whereas the mRNA for M-FCN is expressed mainly in peripheral monocytes and the protein is present in the serum at low concentrations [9]. Two ficolins have been identified in mice: ficolin $\mathrm{A}(\mathrm{FcnA})$ and ficolin $\mathrm{B}(\mathrm{F} \mathrm{cnB})[10,11]$. FcnA mRNA is mainly expressed in Kupffer cells in the liver [12], and the protein is present in serum. FcnB mRNA is mainly expressed in cells of myeloid cell lineage within the bone marrow [12]. The location of $\mathrm{F} \mathrm{cnB}$ protein is still unclear, although it is reported to localize within the lysosomes of activated macrophages [13].
Our phylogenetic analyses show that $\mathrm{FcnB}$ is the murine orthologue of human M-FCN, that FcnA and L-FCN were independently diverged in the murine and primate lineages, respectively, from the ancestral $\mathrm{F} \mathrm{cnB} / \mathrm{M}-\mathrm{FCN}$, and that the $H-F C N$ gene is a pseudogene in the murine lineage [14]. In addition, our previous ontogenetic study showed that the spatial-temporal expression pattern was different for $\mathrm{FcnB}$ and $\mathrm{F} c \mathrm{~A} \mathrm{~A}$, suggesting that each of the ficolins might have a specific role in the prenatal and postnatal stages [12].

Thus, ficolins are roughly classified into two groups: a serum type (plasma type), which includes L-FCN, HFCN and FcnA produced mainly in the liver and present in the circulation as serum lectins [15] and a nonserum type (nonplasma type), which includes $\mathrm{M}-\mathrm{FCN}$ and $\mathrm{F} \mathrm{cnB}$ hardly detectable in the serum. The latter group, particularly murine $\mathrm{FcnB}$, has not been studied in detail at the protein level, because of difficulties in identifying and isolating a sufficient amount of the protein. There are no reports regarding the biochemical features of native $\mathrm{FcnB}$. 
To date, we have shown that mammalian ficolins, including three human ficolins and mouse FcnA, associate with mannose-binding lectin (MBL)-associated serine proteases (MASPs) and activate the lectin pathway [5, 16-18]. We also reported that recombinant mouse $\mathrm{FcnB}$ produced in Drosophila S2 cells does not associate with MASP-2 and small MBL-associated protein (sMAP) [18]. Recently, however, it was reported that rat recombinant $\mathrm{FcnB}$ associates with MASPs and activates the lectin pathway by binding to PAMPs [19]. In the present study, we carefully examined the biochemical properties of FcnB using both native FcnB isolated from mouse bone marrow fluid, and recombinant mouse FcnB produced in $\mathrm{CHO}$ cells. The results show that like rat FcnB, mouse FcnB has the ability to form complexes with MASPs and sMAP.

\section{Materials and Methods}

2.1. Preparation of FcnB from Mouse Bone Marrow. To avoid complications resulting from the co-presence of FcnA and $\mathrm{FcnB}$, the bone marrow tissue used as the source of FcnB was collected from FcnA-deficient mice generated on a C57BL6 background by gene targeting (manuscript in preparation). The bone marrow fluid and cells were collected as supernatant and precipitate, respectively, by centrifugation of the pooled tissue at $10,000 \times \mathrm{g}$ for $10 \mathrm{~min}$. The supernatant was then subjected to affinity chromatography on a GlcNAcagarose column. The bound fraction was eluted sequentially with $0.3 \mathrm{M}$ mannose and then with $0.3 \mathrm{M}$ GlcNAc. The recovered eluate was dialyzed against $50 \mathrm{mM}$ Tris- $\mathrm{HCl}$, at $\mathrm{pH} 7.5$, containing $0.15 \mathrm{M} \mathrm{NaCl}$ and $2.5 \mathrm{mM} \mathrm{CaCl}_{2}$ (TBS$\mathrm{Ca}$ ), concentrated in a centrifugal filter (Amicon Ultra-4, Millipore, Billerica, MA, USA) and stored at $-80^{\circ} \mathrm{C}$ until required. The GlcNAc-eluate was used as a source of native FcnB in further study.

2.2. Preparation of Recombinant FcnB ( $r F c n B)$. Full-length mouse FcnB cDNA was constructed in a pIRCMV vector and cotransfected with a pFerH vector encoding a transposase into $\mathrm{CHO}$ cells as previously described [20]. The rFcnBproducing $\mathrm{CHO}$ cells were screened by culturing in the presence of $0.5 \mathrm{mg} / \mathrm{mL}$ neomycin G-418. After several passages in DMEM medium containing 10\% FCS, the neomycinresistant $\mathrm{CHO}$ cells were cultured in a serum-free medium (CHO-S-SFM, GIBCO, Grand Island, NY, USA). The rFcnB secreted into the culture medium was purified on a GlcNAcagarose column. Briefly, after washing the column with TBS containing $0.05 \%$ Tween-20, $0.1 \mathrm{M}$ mannose, $0.1 \mathrm{M}$ galactose, and $0.1 \mathrm{M}$ glucose, the bound fraction was eluted with $0.3 \mathrm{M}$ GlcNAc. The eluate was dialyzed against TBS$\mathrm{Ca}$, concentrated, and stored at $-80^{\circ} \mathrm{C}$ until use. The $\mathrm{N}$ terminal amino acid sequence of $\mathrm{rFcnB}$ was determined using a Procise cLC Protein Sequencing System (Applied Biosystems, Poster City, CA, USA). The rFcnB produced by $\mathrm{CHO}$ cells was simply termed $\mathrm{rFcnB}$ in this study, while $\mathrm{rFcnB}$ produced previously in Drosophila S2 cells was termed rFcnBs2-1 [18]. The recombinant protein concentration was determined using a BCA protein assay kit (Pierce, Rockford, IL, USA) with BSA as a standard protein.

2.3. Preparation of Recombinant Mouse MASP-1, MASP-2, $M A S P-3$, and $s M A P$. Two recombinant forms of mature mouse MASP-2 were produced in Drosophila S2 cells with a histidine (His)-tag as previously described [21]: one comprised the normal sequence with protease activity (rMASP2a) and the other a mutated sequence (Ser632Ala) with no activity (rMASP-2i). Recombinant mouse MASP-1 (rMASP1i) was prepared in a His-tagged form using a Baculovirus expression system (Invitrogen, Carlsbad, CA, USA), and is an inactive form harboring a mutated catalytic site (Ser646Ala) [22]. Recombinant mouse MASP-3 (rMASP-3) [23] and sMAP (rsMAP) [21] were prepared as His-tagged forms in Drosophila S2 cells. All recombinants (rMASP-1i, -2i, -2a and -3 , and rsMAP) were purified by affinity chromatography on Ni-NTA agarose columns (Qiagen Inc., Valencia, CA, USA) followed by elution with imidazole. The recombinant proteins were dialyzed against TBS, concentrated, and stored at $-80^{\circ} \mathrm{C}$ until use. The protein concentration was determined as described above.

2.4. Western Blotting for FcnB, MBLs, MASPs, and sMAP. SDS-PAGE was performed on $10 \%$ polyacrylamide gels under reducing conditions according to the method of Laemmli. After electrophoresis, the proteins were transferred to a polyvinylidene difluoride membrane filter (Millipore, Billerica, MA, USA). The membrane filter was blocked with Blocking One reagent (Nacalai Tesque Inc., Kyoto, Japan) and probed with 500 2000-fold-diluted polyclonal antibodies (Abs) against mouse FcnB and MASP-2/sMAP [18, 21] and 500-fold-diluted monoclonal Abs against MBL-A and MBLC (clones 8G6 and 16A8, resp., Hycult Biotechnology, Uden, The Netherlands) in $10 \mathrm{mM}$ phosphate buffer, at $\mathrm{pH} 7.4$, containing $137 \mathrm{mM} \mathrm{NaCl}$ and $2.7 \mathrm{mM} \mathrm{KCl}$ (PBS) containing $0.1 \%$ Tween-20 (PBS-T). For the detection of MASP-1 and -3, a monoclonal anti-penta-His-tag Ab (Qiagen) was used as the primary Ab. After washing, the filters were further incubated with either HRP-conjugated secondary Abs or biotinylated secondary Abs (Dako Cytomation, Glostrup, Denmark) followed by an avidin-biotinylated HRP complex (Vector Lab., Burlingame, CA, USA). Finally, the membranes were developed using a chemiluminescent substrate (ECL, Amersham Biosciences, Buckinghamshire, UK). The chemiluminescent image was analyzed using an LAS-3000 (Fuji film, Tokyo, Japan).

2.5. Treatment of FcnB with Endoglycosidases. The N-linked carbohydrates expressed on $\mathrm{FcnB}$ were removed by treatment with endoglycosidase F (EMD Biosciences Inc., La Jolla, CA, USA) as previously described [24]. Selective removal of O-linked glycans was achieved by treatment with $0.1 \mathrm{U}$ neuraminidase (Wako Pure Chemicals, Osaka, Japan) and $20 \mathrm{mU}$ endo- $\alpha-N$-acetylgalactosaminidase (Seikagaku Co., Tokyo, Japan) at $37^{\circ} \mathrm{C}$ for $16 \mathrm{~h}$. 
2.6. Gel Filtration Chromatography of FcnB. To estimate the size distribution of oligomeric FcnB, the F $\mathrm{cnB}$ preparations were subjected to gel filtration chromatography using a Superose 6 10/300GL column equilibrated with PBS and connected to an ÄKTA purifier system (Amersham Biosciences, Uppsala, Sweden). An aliquot of each recovered fraction $(0.5 \mathrm{~mL} /$ fraction) was assessed for FcnB by western blotting under reducing conditions.

2.7. Complex Formation of $r F c n B$ with $r M A S P s$ and $r s M A P$. $\mathrm{rFcnB}$ was incubated with rMASPs and rsMAP at a molar ratio of $3: 1: 8(\mathrm{rFcnB}: \mathrm{rMASP}: \mathrm{rsMAP})$ overnight at $4^{\circ} \mathrm{C}$ in TBS containing $2.5 \mathrm{mM} \mathrm{CaCl}_{2}, 3 \% \mathrm{BSA}$, and $0.05 \%$ Tween20 as previously described [18]. The above molar ratio was chosen by reference to the concentrations of FcnA, MASP-2, and sMAP in the mouse serum [18]. The mixture was further incubated with a GlcNAc-agarose slurry $(50 \%, 40 \mu \mathrm{L})$ at $4^{\circ} \mathrm{C}$ for $3 \mathrm{hr}$ to pull down $\mathrm{rFcnB}$, and the bound fraction was eluted with $0.3 \mathrm{M}$ GlcNAc. The eluate was dialyzed against TBS-Ca and the final sample subjected to western blotting and a C4-deposition assay. For western blotting, rMASP-2i was used as a source of MASP-2 to ensure clear results, since it is known that rMASP-2a is converted, in part, into its active form, comprising the heavy and light chains connected via a disulfide bond, during purification. For the C4-deposition assay, MASP-2a was used as the source of MASP-2 instead of rMASP-2i. Similar autoactivation is also seen with rMASP-1; therefore, rMASP-1i was used as the source of MASP-1 for western blotting to detect complex formation with $\mathrm{rFcnB}$.

2.8. C4-Deposition Assay. C4-deposition activity was determined by ELISA as previously described [18]. Briefly, the GlcNAc eluates prepared from bone marrow fluid or the $\mathrm{rFcnB} / \mathrm{rMASP}-2 \mathrm{a} / \mathrm{sMAP}$ complexes, were incubated in $100 \mu \mathrm{L}$ of TBS-Ca at $37^{\circ} \mathrm{C}$ for $10 \mathrm{~min}$ in a GlcNAc-BSAcoated microtiter plate. The plate was then incubated with human $\mathrm{C} 4$ on ice for $30 \mathrm{~min}$, followed by washing with PBS-T. The C4b generated on the plate was detected with an HRP-conjugated sheep anti-human $\mathrm{C} 4 \mathrm{~b} \mathrm{Ab}$ (Biogenesis, Poole, UK) and color developed using TMB (KPL Co., Gaithersburg, MD, USA) and $\mathrm{H}_{2} \mathrm{O}_{2}$ as substrates. After termination of the reaction with $0.5 \mathrm{M} \mathrm{H}_{3} \mathrm{PO}_{4}$, the plates were read at $450 \mathrm{~nm}$ in a Multimode detector DTX880 (Beckman Coulter Inc., Brea, CA, USA).

\section{Results}

To detect the FcnB protein in the bone marrow, the tissue supernatants and precipitates were subjected to western blotting. As shown in Figure $1(\mathrm{a})$, a $38 \mathrm{kDa}$ band was observed in the supernatant under reducing conditions, suggesting that $\mathrm{FcnB}$ is secreted into the mouse bone marrow fluid as a soluble protein. $\mathrm{FcnB}$ was also detected as a $37 \mathrm{kDa}$ band at high levels in the precipitate. This suggests that FenB in bone marrow cells is slightly small due to incomplete processing prior to secretion. When FcnB in the supernatant was treated with endoglycosidase $\mathrm{F}$, its molecular weight

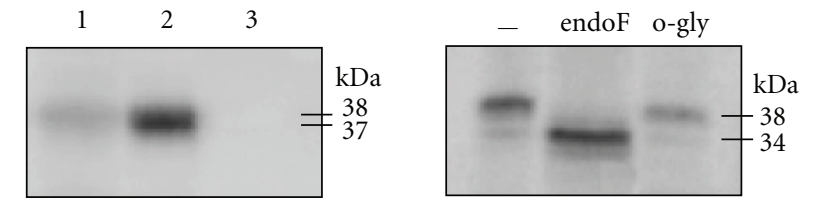

(a)

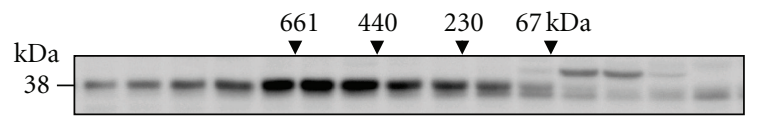

(b)

Figure 1: Characterization of FcnB in the mouse bone marrow. (a) Western blotting of F $\mathrm{cnB}$ in the supernatant (lane 1) and precipitate (lane 2) of bone marrow tissue, and plasma (lane 3) (left panel). Two $\mu \mathrm{L}$ of the supernatant/precipitate equivalent to the original tissue and $2 \mu \mathrm{L}$ of plasma from $\mathrm{FcnA}^{-/-}$mice were subjected to western blotting under reducing conditions. FcnB in the supernatant ( $4 \mu \mathrm{L}$ equivalent to the original tissue) was treated with endoglycosidase $\mathrm{F}$ (endo $F$ ) or neuraminidase plus endo- $\alpha-\mathrm{N}$ acetylgalactosaminidase (o-gly) and subjected to western blotting (right panel).-, not treated. (b) Superose 6 gel chromatography of $\mathrm{FcnB}$ in the bone marrow supernatant from $\mathrm{FcnA}^{-/-}$mice. An aliquot of each fraction was subjected to western blotting under reducing conditions. Arrowheads depict the eluted positions of the molecular weight markers $(661 \mathrm{kDa}$, thyroglobulin; $440 \mathrm{kDa}$, ferritin; $230 \mathrm{kDa}$, catalase; $67 \mathrm{kDa}, \mathrm{BSA})$.

reduced from 38 to $34 \mathrm{kDa}$, whereas treatment with endo- $\alpha$ $\mathrm{N}$-acetylgalactosaminidase resulted in either no or a smaller reduction in molecular weight (Figure 1(a)). To determine the size distribution of oligomeric FcnB, the supernatant was subjected to gel chromatography. FcnB was recovered from fractions corresponding to the elution positions of marker proteins ranging from 100 to $>1000 \mathrm{kDa}$ with a peak around $600 \mathrm{kDa}$ (Figure 1(b)), indicating a heterogeneous structure composed mainly of $12-18$-mers.

Next, the bone marrow supernatant was subjected to GlcNAc-agarose affinity chromatography to purify FcnB. As shown in Figure $2(\mathrm{a}), \mathrm{FcnB}$ was recovered in the GlcNAc eluate, whereas MBL-A and MBL-C (MBLs) were recovered in the mannose eluate. The mannose eluate included significant amounts of the MASP-2 pro-enzyme, MASP-2 heavy chain, and sMAP. This suggests that MBLs are present in the bone marrow fluid as complexes with MASP-2 and sMAP. Interestingly, trace amounts of MASP-2 and sMAP were also detected in the GlcNAc eluate, suggesting that at least a part of FenB also exists in complex with MASP-2 and sMAP. This GlcNAc eluate showed C4-deposition on GlcNAc-coated microplates (Figure 2(b)), although the level was very low compared with that of the mannose eluate. This activity was significantly decreased by passage of the eluate through anti-FcnB Ab-coupled Sepharose 4B. These results suggest that the $\mathrm{FcnB} / \mathrm{MASP}$ complexes can activate complement component $\mathrm{C} 4$ through the lectin pathway.

To confirm the above results, $\mathrm{rFcnB}$ was produced in $\mathrm{CHO}$ cells and purified by GlcNAc-agarose chromatography. Western blotting showed that $\mathrm{rFcnB}$ consisted of a monomer with a molecular weight of $37 \mathrm{kDa}$; slightly smaller 


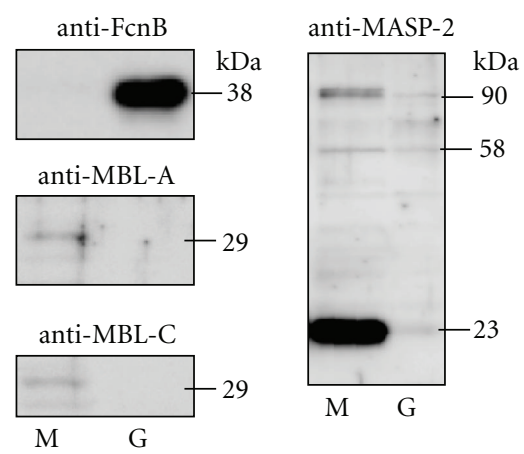

(a)

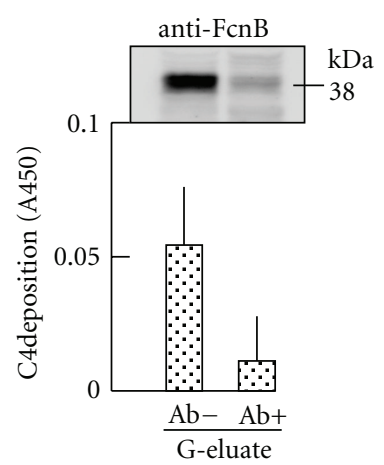

(b)

Figure 2: : GlcNAc-agarose chromatography of bone marrow fluids from FcnA ${ }^{-/}$mice. (a) Western blot of FcnB, MBL-A, MBL-C, and MASP-2/sMAP in the mannose $(\mathrm{M})$ and GlcNAc $(\mathrm{G})$ eluates from GlcNAc-agarose chromatography under reducing conditions. Right panel: $90 \mathrm{kDa}, 58 \mathrm{kDa}$, and $23 \mathrm{kDa}$ bands represent the proenzyme form of MASP-2, the heavy chain of MASP-2, and sMAP, respectively. For each sample, $60 \mu \mathrm{L}$ equivalent to the original volume of bone marrow tissue was loaded per lane. (b) C4-deposition activity of the GlcNAc eluate on GlcNAc-BSA-coated microplates. Before assessment, the GlcNAc eluate was passed through anti-FcnB Ab-coupled Sepharose 4B (Ab+) or not $(\mathrm{Ab}-)$. The activity of a $30 \mu \mathrm{L}$ sample equivalent to the original bone marrow tissue was determined in quadruplicate (mean $\pm \mathrm{SD}$ ). Inset: western blot of $\mathrm{FcnB}$ in the eluates used for $\mathrm{C} 4$ deposition.
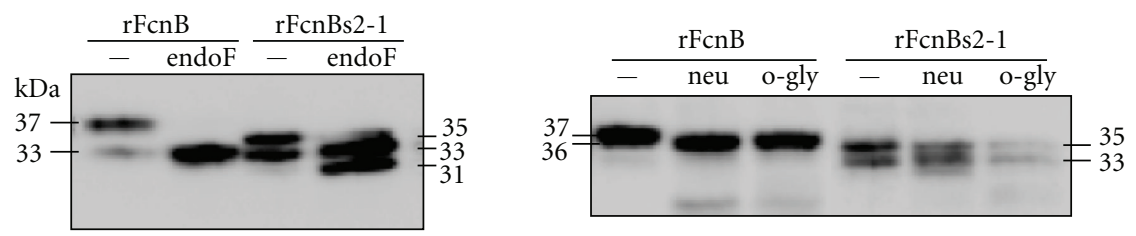

(a)

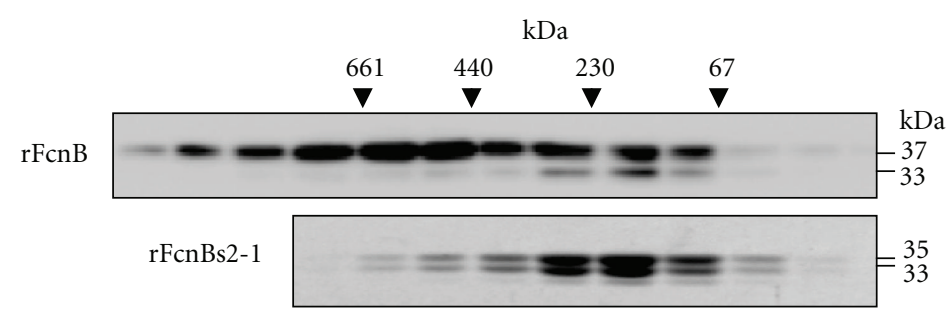

(b)

FIgURE 3: Structural characterization of $\mathrm{rFcnB}$. (a) Left panel: Western blot of $\mathrm{rFcnB}$ and $\mathrm{rFcnBs} 2-1$ treated with endoglycosidase F (endoF). Right panel: western blot of $\mathrm{rFcnB}$ and $\mathrm{rFcnBs} 2-1$ treated with neuraminidase (neu) or neuraminidase plus endo- $\alpha-N$ acetylgalactosaminidase (o-gly). Western blotting was performed under reducing conditions. —, not treated. (b) Gel chromatography of $\mathrm{rFcnB}$ (upper panel) and $\mathrm{rFcnBs} 2-1$ (lower panel). $\mathrm{rFcnB}$ or $\mathrm{rFcnBs} 2-1(400 \mu \mathrm{L} ; 5-10 \mu \mathrm{g})$ was applied to a Superose $6 \mathrm{column}(1 \mathrm{~cm} \phi \times$ $30 \mathrm{~cm}$ ) and fractionated into $0.5 \mathrm{~mL} /$ fractions. An aliquot of each fraction was subjected to western blotting for FcnB under reducing conditions.

than native FcnB, but larger than $\mathrm{rFcnBs} 2-1(33-35 \mathrm{kDa})$ (Figure 3(a)). Upon treatment with endoglycosidase F, the molecular weights of $\mathrm{rFcnB}$ and $\mathrm{rFcnBs} 2-1$ were reduced to $33 \mathrm{kDa}$ and $31-33 \mathrm{kDa}$, respectively. Treatment of $\mathrm{rFcnB}$ with endo- $\alpha$ - $\mathrm{N}$-acetylgalactosaminidase resulted in a slight reduction in the molecular weight to $36 \mathrm{kDa}$, while treatment of $r F c n B s 2-1$ had no effect. The $\mathrm{N}$-terminal amino acid sequence of $\mathrm{rFcnB}$ was $\mathrm{T}^{20} \mathrm{CPELKV}$, indicating that the preceding 19 amino acids were removed as a signal peptide by the host $\mathrm{CHO}$ cells. The N-terminal sequences of the $35 \mathrm{kDa}$ and $33 \mathrm{kDa}$ bands of $\mathrm{rFcnBs} 2-1$ were RSPWPGVFV ${ }^{15} \mathrm{HAAG}$ and $\mathrm{A}^{18} \mathrm{GTCPEL}$, respectively, indicating that the $35 \mathrm{kDa}$ band corresponded to our designed $\mathrm{rFcnBs} 2-1$ product containing the eight plasmid-derived amino acids (underlined) at the N-terminal [18], and the $33 \mathrm{kDa}$ band was another rFcnBs2-1 product with a different $\mathrm{N}$-terminal, which was processed by Drosophila S2 cells.

Oligomeric $\mathrm{rFcnB}$ was subjected to gel chromatography to determine its size distribution. It was found that, like native $\mathrm{FcnB}$, the main $\mathrm{rFcnB}$ species was eluted in the 

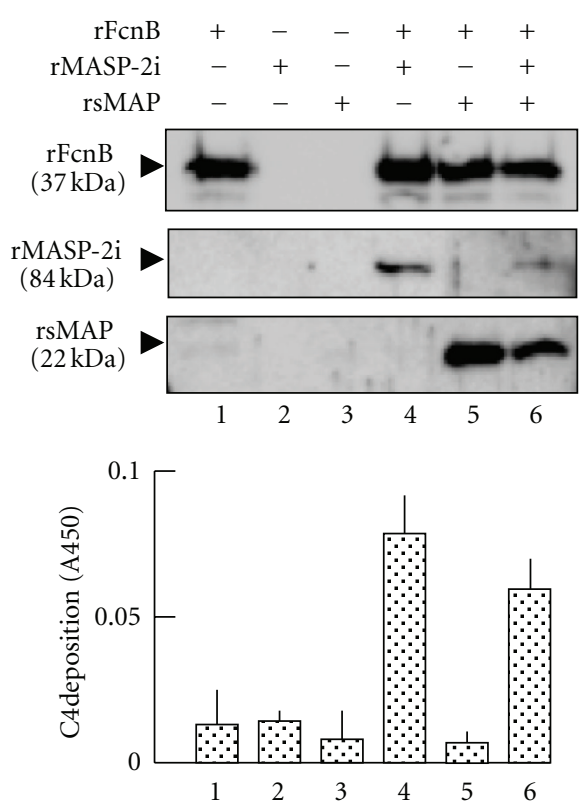

Figure 4: Complement activation by $\mathrm{rFcnB} / \mathrm{rMASP}-2 / \mathrm{rsMAP}$ complexes. Upper panel: western blot of FcnB and MASP-2/sMAP. After incubation of the recombinant proteins as shown in the table, the generated complexes were pulled down by GlcNAc-agarose and subjected to western blotting. Lower panel: C4-deposition activity of the similar pull-down samples on GlcNAc-BSA-coated microplates. The samples used for $\mathrm{C} 4$ deposition were prepared the same as those for western blotting except for employment of rMASP-2a instead of rMASP-2i. The activity was determined in quadruplicate (mean $\pm \mathrm{SD}$ ).

range corresponding to 100 to $>1000 \mathrm{kDa}$ with a peak around $600 \mathrm{kDa}$ (Figure 3(b)). A minor band was observed at $33 \mathrm{kDa}$ in the $\mathrm{rFcnB}$ preparation eluted between 100 and $200 \mathrm{kDa}$. These results indicate that the $\mathrm{rFcnB}$ preparation contains a major 12-18-mer made up of $37 \mathrm{kDa}$ monomers, and a minor 3-6-mer made up of $33 \mathrm{kDa}$ monomers. Gel chromatography of $\mathrm{rFcnBs} 2-1$ showed that this protein ranged from 100 to $300 \mathrm{kDa}$, suggesting that it is a 3-9-mer composed of 33-35 kDa monomers.

To confirm the interaction between $\mathrm{rFcnB}$ and MASPs and sMAP, rFcnB was incubated with rMASP-2i and rsMAP and then subjected to FcnB pull down using GlcNAcagarose. As shown in Figure 4, rMASP-2i and rsMAP were coprecipitated only in the presence of $\mathrm{rFcnB}$. Coincubation of rMASP-2 $\mathrm{i}$ and rsMAP resulted in reduced binding to $\mathrm{rFcnB}$ compared with incubation with each alone, suggesting their competitive bindings to $\mathrm{rFcnB}$. The $\mathrm{rFcnB} / \mathrm{rMASP}-$ 2a complex activated $\mathrm{C} 4$ on GlcNAc-coated microplates. This C4-deposition activity was inhibited in part by rsMAP. In addition, $r F c n B$ bound to rMASP-1 $i$ and rMASP-3, and this binding was partially inhibited by coincubation with rsMAP (Figures 5(a) and 5(b)), suggesting that FcnB associates with all types of MASP and sMAP in a similar manner. No activation of rMASP-3 was observed under these experimental conditions, even when it was complexed with $\mathrm{rFcnB}$ on GlcNAc.

\section{Discussion}

The present study clearly indicates that, like rat recombinant $\mathrm{F} c \mathrm{BB}$, both native and recombinant forms of mouse $\mathrm{FcnB}$ associate with MASPs and sMAP. It also demonstrates that FcnB/MASPs/sMAP complexes activate $\mathrm{C} 4$ on immobilized GlcNAc. Taken together with the results of Girija et al. [19], these results suggest that at least a part of murine FcnB essentially executes its function through the lectin pathway.

In the present study, it was observed that the monomer size of FcnBs2-1 was smaller than that of the native FcnB and $\mathrm{rFcnB}$ proteins, largely due to the $\mathrm{N}$-linked carbohydrate content. It was also found that FcnBs2-1 formed smaller oligomers (3-9-mers), in contrast to the highly oligomeric forms of the native $\mathrm{FcnB}$ and $\mathrm{rFcnB}$ (12-18-mers). These results simply suggest that the processing of proteins in insect cells is different from that in mammalian cells. To confirm this in the present study, we prepared a third form of recombinant mouse FcnB, termed rFcnBs2-2, in Drosophila $\mathrm{S} 2$ cells. The $\mathrm{N}$-terminal residue of $\mathrm{rFcnBs} 2-2$ was adjusted to $\mathrm{Thr}^{20}$ as same as that in $\mathrm{rFcnB}$, which was performed by ligation of a F cnB cDNA containing an extra six bases into a pMT/Bip/V5-His A vector. The generated rFcnBs2-2 showed a molecular weight of $31 \mathrm{kDa}$ under reducing conditions, and treatment with endoglycosidase F reduced this to $30 \mathrm{kDa}$ (data not shown). It was also found that rFcnBs2-2 was less oligomeric, existing mainly as 3-6-mers, and that it failed to associate with rMASP-2i or rsMAP (data not shown). These results clearly indicate that the processing of recombinant mouse FcnB in Drosophila S2 cells is different from that in mammalian cells.

An interesting result was observed in gel chromatography of the $\mathrm{rFcnB}$ preparation, which contained a major and highly oligomeric species comprising $37 \mathrm{kDa}$ monomers and a minor and poorly oligomeric species comprising $33 \mathrm{kDa}$ monomers (Figure 3(b)). In a preliminary study, we observed that culture of $\mathrm{CHO}$ cells in the presence of tunicamycin, an inhibitor of N-linked glycosylation, resulted in the preferential production of $\mathrm{rFcnB}$ comprising $33 \mathrm{kDa}$-monomers, which was also less oligomeric (3-6mers) (data not shown). These results suggest that full $\mathrm{N}$ linked glycosylation of FcnB is essential for high level of oligomerization.

We recently observed that Drosophila S2 cells produced trimers of human $\mathrm{H}-\mathrm{FCN}$, while $\mathrm{CHO}$ cells produced highly oligomeric H-FCN ( 18-mers), which were structurally similar to native H-FCN in human serum (data not shown). However, Drosophila S2 cells do not always produce less oligomeric ficolin. For example, this cell line successfully produced highly oligomeric forms of mouse FcnA and human M-FCN ( $\sim 600 \mathrm{kDa})[17,18]$. At present, the reason why ficolin molecules are processed differently in Drosophila $\mathrm{S} 2$ cells it is not known. One possibility might be a small difference among these ficolins in the amino acid sequence that forms the $\mathrm{N}$-linked glycosylation site, for example, the surrounding sequence around Asn-X-Ser/Thr.

Our studies, including the present study, demonstrate that, regardless of the type of host cell used, recombinant ficolins expressed as highly oligomeric forms associate with 


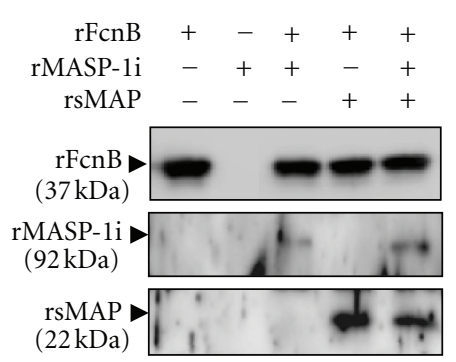

(a)

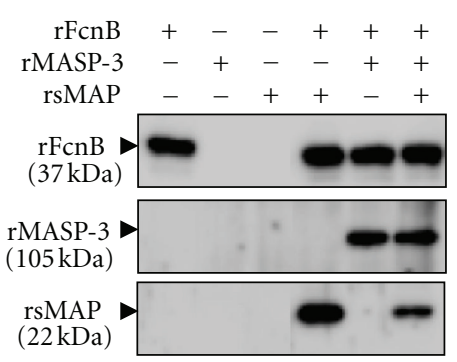

(b)

FIgURE 5: Complex formation between rFcnB and rMASP-1i (a) and rMASP-3 (b). After incubation of the recombinant proteins as outlined in the table, samples pulled down with GlcNAc agarose were subjected to western blotting. Western blotting for rMASP-1i, rMASP-3, and rsMAP was performed using an anti-penta-His tag $\mathrm{Ab}$.

MASPs/sMAP, while less oligomeric forms do not. Thus, it is suggested that full processing including N-linked glycosylation is essential for the formation of highly oligomeric ficolin and that high oligomerization is in turn essential for association with MASPs and sMAP. At least, it can be concluded from our studies that $\mathrm{CHO}$ cells are available to produce highly oligomeric recombinant for all types of ficolin.

The molecular mechanism underlying the activation of the lectin pathway by mouse $\mathrm{FcnB}$ appears to be similar to that of other mammalian ficolins, at least in terms of complex formation with MASPs, sMAP, and probably Map44 (MAP-1) [25] and in activating C4, possibly C3 and C2 on targets. However, it is unclear as to how strongly FcnB exerts its activity through the lectin pathway in vivo and as to how FenB shares its activity with FcnA and MBLs. It is noteworthy that, in the present study, rMASP-3 was not activated when complexed with $\mathrm{rFcnB}$ on GlcNAc. The $\mathrm{rFcnB} / \mathrm{rMASP}-3$ complex may be useful for identifying native target molecule(s) recognized by FcnB.

\section{Conclusions}

FcnB was identified in mouse bone marrow fluid as a highly oligomeric protein. GlcNAc-agarose chromatography of bone marrow fluid showed that $\mathrm{FcnB}$ was present in a complex with MASP-2 and sMAP. This complex also exhibited C4-deposition activity on immobilized GlcNAc. These results were confirmed using $\mathrm{rFcnB}$ produced in $\mathrm{CHO}$ cells. Comparison of the biochemical features of the three types of the recombinant ficolin, $\mathrm{rFcnB}, \mathrm{rFcnBs} 2-1$, and rFcnBs2-2, suggests that full processing, including $\mathrm{N}$-linked glycosylation, is essential for oligomerization of $\mathrm{FcnB}$ and its association with MASPs/sMAP. Taken together, we conclude that mouse $\mathrm{F} c \mathrm{BB}$ acts as a recognition molecule working through the lectin pathway. To further understand the role of murine $\mathrm{FcnB}$, its location site and real target(s) and the precise stoichiometry of the FcnB complex need to be clarified.

\section{Acknowledgments}

The authors thank Ms N. Nakazawa and K. Kanno for technical assistance. This work was supported in part by grants from the Ministry of Education, Culture, Sports, Science and Technology of Japan.

\section{References}

[1] M. Matsushita, Y. Endo, S. Taira et al., "A novel human serum lectin with collagen- and fibrinogen-like domains that functions as an opsonin," Journal of Biological Chemistry, vol. 271, no. 5, pp. 2448-2454, 1996.

[2] V. Garlatti, N. Belloy, L. Martin et al., "Structural insights into the innate immune recognition specificities of $\mathrm{L}$ - and $\mathrm{H}$ ficolins," EMBO Journal, vol. 26, no. 2, pp. 623-633, 2007.

[3] V. Garlatti, L. Martin, E. Gout et al., "Structural basis for innate immune sensing by M-ficolin and its control by a pH-dependent conformational switch," Journal of Biological Chemistry, vol. 282, no. 49, pp. 35814-35820, 2007.

[4] M. Tanio, S. Kondo, S. Sugio, and T. Kohno, "Trivalent recognition unit of innate immunity system: crystal structure of trimeric human M-ficolin fibrinogen-like domain," Journal of Biological Chemistry, vol. 282, no. 6, pp. 3889-3895, 2007.

[5] M. Matsushita, Y. Endo, and T. Fujita, "Complementactivating complex of ficolin and mannose-binding lectinassociated serine protease," Journal of Immunology, vol. 164, no. 5, pp. 2281-2284, 2000.

[6] Y. Endo, Y. Sato, M. Matsushita, and T. Fujita, "Cloning and characterization of the human lectin P35 gene and its related gene," Genomics, vol. 36, no. 3, pp. 515-521, 1996.

[7] J. Lu, P. N. Tay, O. L. Kon, and K. B. M. Reid, "Human ficolin: cDNA cloning, demonstration of peripheral blood leucocytes as the major site of synthesis and assignment of the gene to chromosome 9," Biochemical Journal, vol. 313, no. 2, pp. 473478, 1996.

[8] R. Sugimoto, Y. Yae, M. Akaiwa et al., "Cloning and characterization of the Hakata antigen, a member of the ficolin/opsonin p35 lectin family," Journal of Biological Chemistry, vol. 273, no. 33, pp. 20721-20727, 1998.

[9] C. Honoré, S. Rørvig, L. Munthe-Fog et al., "The innate pattern recognition molecule Ficolin-1 is secreted by monocytes/macrophages and is circulating in human plasma," Molecular Immunology, vol. 45, no. 10, pp. 2782-2789, 2008.

[10] Y. Fujimori, S. Harumiya, Y. Fukumoto et al., "Molecular cloning and characterization of mouse ficolin-A," Biochemical and Biophysical Research Communications, vol. 244, no. 3, pp. 796-800, 1998.

[11] T. Ohashi and H. P. Erickson, "Oligomeric structure and tissue distribution of ficolins from mouse, pig and human," Archives 
of Biochemistry and Biophysics, vol. 360, no. 2, pp. 223-232, 1998.

[12] Y. Liu, Y. Endo, S. Homma, K. Kanno, H. Yaginuma, and T. Fujita, "Ficolin A and ficolin B are expressed in distinct ontogenic patterns and cell types in the mouse," Molecular Immunology, vol. 42, no. 11, pp. 1265-1273, 2005.

[13] V. L. Runza, T. Hehlgans, B. Echtenacher, U. Zähringer, W. J. Schwaeble, and D. N. Männel, "Localization of the mouse defense lectin ficolin B in lysosomes of activated macrophages," Journal of Endotoxin Research, vol. 12, no. 2, pp. 120-126, 2006.

[14] Y. Endo, Y. Liu, K. Kanno, M. Takahashi, M. Matsushita, and T. Fujita, "Identification of the mouse $\mathrm{H}$-ficolin gene as a pseudogene and orthology between mouse ficolins A/B and human L-/M-ficolins," Genomics, vol. 84, no. 4, pp. 737-744, 2004.

[15] Y. Endo, M. Matsushita, and T. Fujita, "Role of ficolin in innate immunity and its molecular basis," Immunobiology, vol. 212, no. 4-5, pp. 371-379, 2007.

[16] M. Matsushita, M. Kuraya, N. Hamasaki, M. Tsujimura, H. Shiraki, and T. Fujita, "Activation of the lectin complement pathway by H-ficolin (Hakata antigen)," Journal of Immunology, vol. 168, no. 7, pp. 3502-3506, 2002.

[17] Y. Liu, Y. Endo, D. Iwaki et al., "Human M-ficolin is a secretory protein that activates the lectin complement pathway," Journal of Immunology, vol. 175, no. 5, pp. 3150-3156, 2005.

[18] Y. Endo, N. Nakazawa, Y. Liu et al., "Carbohydrate-binding specificities of mouse ficolin A, a splicing variant of ficolin A and ficolin B and their complex formation with MASP-2 and sMAP," Immunogenetics, vol. 57, no. 11, pp. 837-844, 2005.

[19] U. V. Girija, D. A. Mitchell, S. Roscher, and R. Wallis, "Carbohydrate recognition and complement activation by rat ficolin-B," European Journal of Immunology, vol. 41, no. 1, pp. 214-223, 2011.

[20] H. Nakanishi, Y. Higuchi, S. Kawakami, F. Yamashita, and M. Hashida, "PiggyBac transposon-mediated long-term gene expression in mice," Molecular Therapy, vol. 18, no. 4, pp. 707$714,2010$.

[21] D. Iwaki, K. Kanno, M. Takahashi et al., "Small mannosebinding lectin-associated protein plays a regulatory role in the lectin complement pathway," Journal of Immunology, vol. 177, no. 12, pp. 8626-8632, 2006.

[22] M. Takahashi, Y. Ishida, D. Iwaki et al., "Essential role of mannose-binding lectin-associated serine protease-1 in activation of the complement factor D," Journal of Experimental Medicine, vol. 207, no. 1, pp. 29-37, 2010.

[23] D. Iwaki, K. Kanno, M. Takahashi, Y. Endo, M. Matsushita, and T. Fujita, "Mannose-binding lectin-associated serine protein 3 (MASP-3) induces activation of the alternative complement pathway," The Journal of Immunology, vol. 187, pp. 3751-3758, 2011.

[24] N. Okada, R. Harada, T. Fujita, and H. Okada, "A novel membrane glycoprotein capable of inhibiting membrane attack by homologous complement," International Immunology, vol. 1, no. 2, pp. 205-208, 1989.

[25] S. E. Degn, A. G. Hansen, R. Steffensen, C. Jacobsen, J. C. Jensenius, and S. Thiel, "MAp44, a human protein associated with pattern recognition molecules of the complement system and regulating the lectin pathway of complement activation," Journal of Immunology, vol. 183, no. 11, pp. 7371-7378, 2009. 

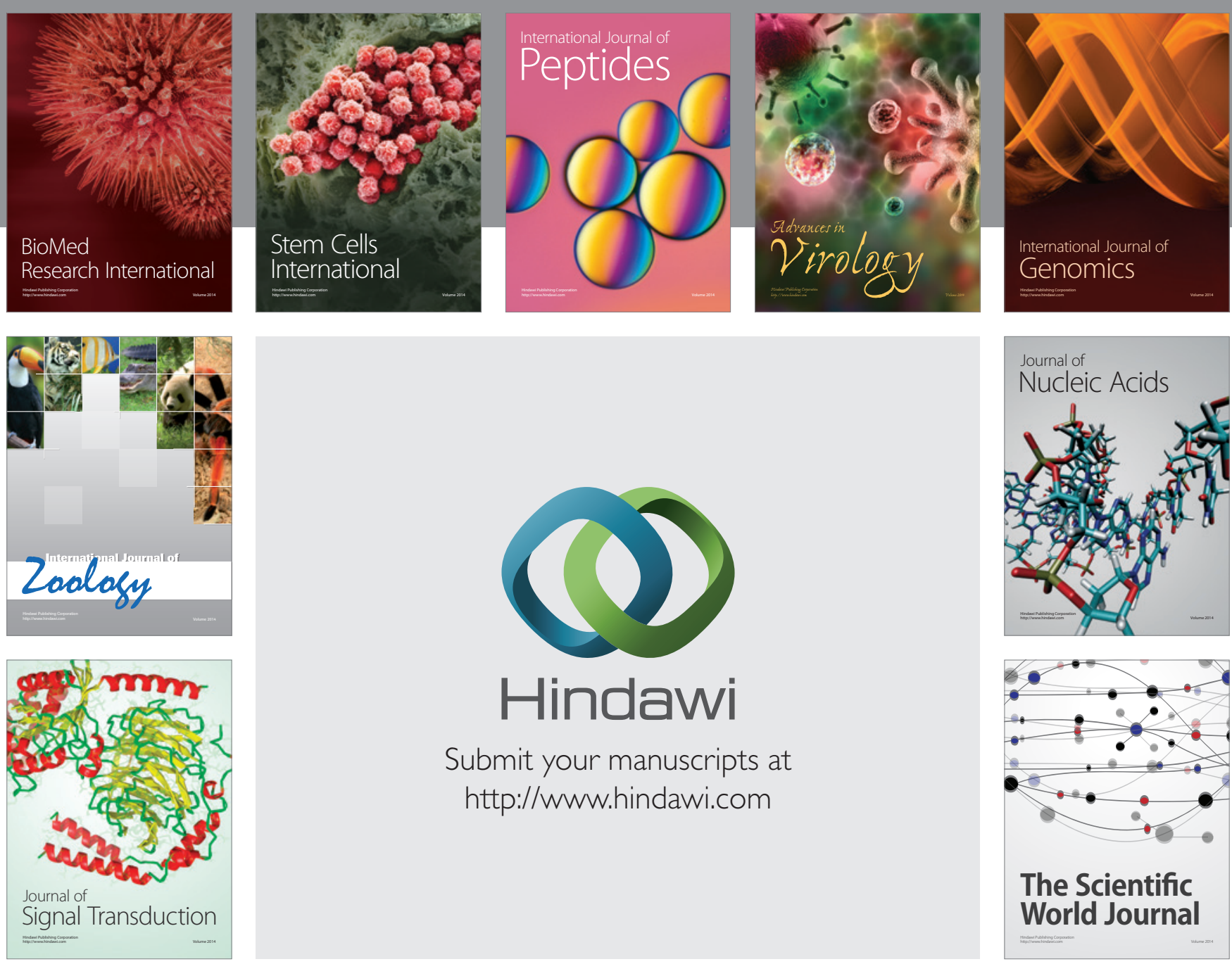

Submit your manuscripts at

http://www.hindawi.com
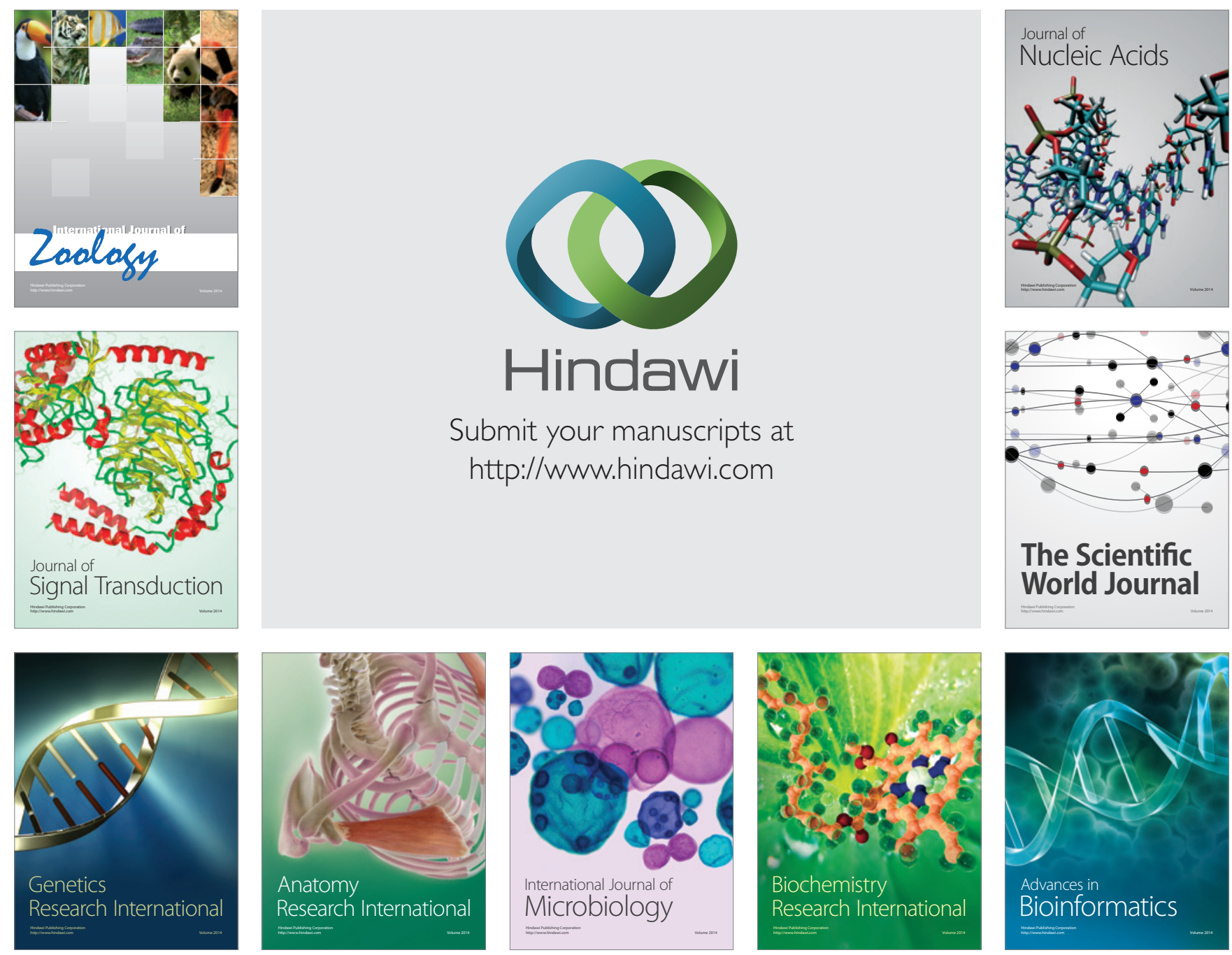

The Scientific World Journal
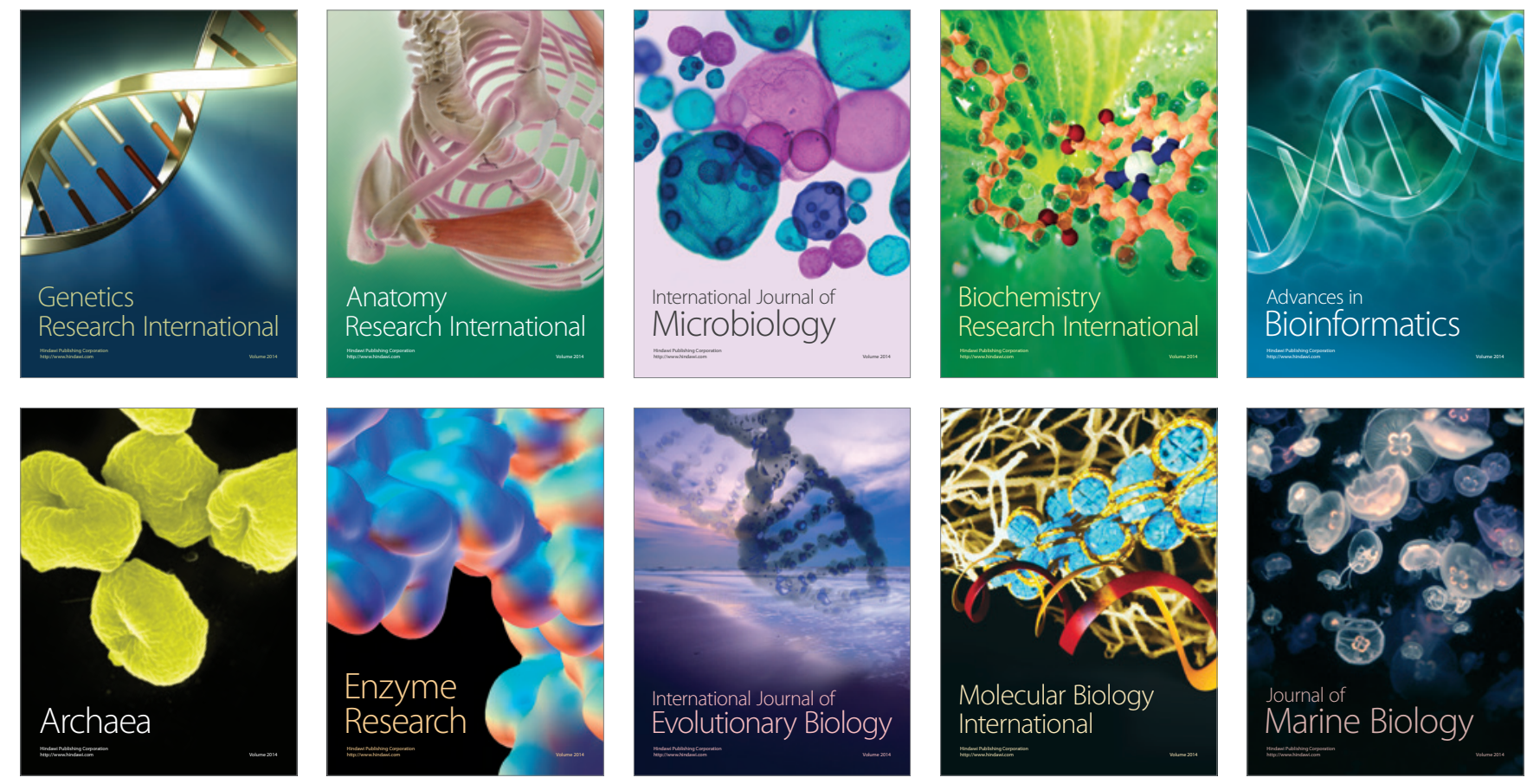